\title{
COVID-19 RISK CALCULATOR
}

A PREPRINT

\author{
Tomás Echavarría \\ Department of Computer Science \\ EAFIT University \\ tomasechavarriab@gmail.com
}

\author{
David Betancur \\ Department of Computer Science \\ EAFIT University \\ david.bet. san@gmail.com
}

\author{
Miguel A. Correa-Manrique \\ Department of Computer Science \\ EAFIT University \\ macorream@eafit.edu.co
}

\author{
Mauricio Toro \\ Department of Computer Science \\ EAFIT University \\ mtorobe@eafit.edu.co
}

\author{
Tomás Olarte \\ Department of Computer Science \\ EAFIT University \\ tolarteh@eafit.edu.co
}

October 1, 2021

\begin{abstract}
COVID-19 has been studied broadly and it has shown a very variant response depending on each individual, there are some factors that can make the disease more or less complex on someone infected. Machine learning models as decision trees have been used for short term prediction of mortality for different conditions such as chronic obstructive pulmonary disease. Vulnerability indexes are important during a pandemic to take especial care of the people who are more susceptible to worst outcomes such as death in case of infection. They are also important so the healthcare system can estimate the installed capacity beforehand and prepare for the upcoming infected patients. This project was designed for use by people in Latin America, especially in Colombia, the data used in this study was taken from Mexico's Open Data from the General Directorate of Epidemiology. This article presents a machine learning model based on decision trees ensembles to predict the probability of someone dying because of the infection, using variables such as comorbidities, sex, gender and other individual conditions. The type of decision tree used was a LightGBM with Bayesian parameter optimization, subsequently calibrated using a sigmoidal function. The importance of each of the variables for the model was evaluated.The performance of the model was evaluated using the AUC ROC, and a result of 0.89 was obtained. This model was later used to develop a mortality calculator to assist health care workers and individuals in making decisions that can help them during the COVID-19 pandemic.
\end{abstract}

Keywords COVID-19 $\cdot$ Ensemble $\cdot$ Machine learning $\cdot$ Decision Tree $\cdot$ Coronavirus $\cdot$ Risk Calculator

\section{Introduction}

COVID-19 (coronavirus disease 2019) first appeared in December 2019 in Wuhan, China, due to a virus called SARSCoV-2 that quickly spread around the world. Accordingly to WHO, it is highly contagious, it's mostly transmitted from person to person through particles originated from the infected person's respiratory system, but it can also be transmitted by touching contaminated surfaces and then the eyes, mouth or nose. By 23 December 2020, there were a total of 94963847 confirmed cases and 2050857 confirmed deaths. The most common COVID-19 symptoms accordingly to WHO are fever, dry cough and fatigue, but there are some other less common symptoms like: loss of taste or smell, nasal congestion, conjunctivitis, sore throat, headache, muscle or joint pain, skin rashes, nausea or vomiting, diarrhea, chills or dizziness. Sever cases include: shortness of breath, loss of appetite, confusion, persistent pain or pressure in the chest, high temperature (above $38{ }^{\circ} \mathrm{C}$ ). Although the global mortality risk of COVID-19 is approximately $2.3 \%$, it increases with age and those with underlying medical problems like heart disease, chronic kidney disease, type 2 diabetes, chronic obstructive pulmonary disease, obesity, or people who have a weak immune 
system. WHO suggests from COVID-19 infected people, $80 \%$ are mild or asymptomatic, $15 \%$ are severe and 5\% are critical infections. Severe and critical cases require hospital care, some of them need intubation, oxygenation or more specialized care. In Colombia, by 23 December 2020, according to the Ministry of Health, the confirmed cases were 1 530593 confirmed cases, with 40931 deaths. Previous studies have been conducted to identify a risk scores in patients diagnosed with different diseases [2], in the specific case of COVID-19, studies have been carried out to develop similar calculators. [4][5]|[1]

It is of great importance to have a risk calculator with which can give meaningful information that can help optimize patient care so the healthcare system can estimate the installed capacity beforehand and prepare for the upcoming infected patients. This article presents a machine learning model based on decision trees ensembles to predict the probability of someones death given the infection using variables such as comorbidities, sex, gender and other individual conditions.

\section{Methods}

\subsection{Data Sources and Processing}

This study is part of EAFIT university's MathCOVID project, which aims to collect, visualize, analyze, predict and evaluate disease control strategies produce by SARS-CoV-2 using mathematical modeling, simulation and artificial intelligence tools.

The data used in this study was taken from Mexico's Open Data from the General Directorate of Epidemiology. A total of 170108 cases of confirmed COVID-19 in Mexico were used for the study, ages from 0 to 120 years, information taken between January and June 2020 by the Health Secretary of Mexico government.

To carry out this project, use was made of information related to the cases of Mexico, since its population is of Latin origin, as well as the population of Colombia, in addition to having access to information on many confirmed cases and in a very detailed manner that included comorbidities and additional conditions.

\subsection{Data Collection and Understanding}

The data obtained from each patient through the open data from Mexico can be divided by categories. The data related to registration information, such as date of update in the system, registration id, date of entry in the database, date of symptoms, date of death. The demographic data are origin of the infection, sector to which the person belongs, the state, sex, national entity, municipality of residence, type of patient, age, nationality, ethnicity, country of nationality, country of origin. The data related to clinical history are pneumonia, pregnancy, asthma, intubation, diabetes, chronic obstructive pulmonary disease, immunosuppression, hypertension, cardiovascular, obesity, chronic renal, smoking, ICU. Variables related to patients medical history, age and clinical conditions give important information about how patient could respond to a COVID-19 infection.

\subsection{Outcome}

This study aims to predict the probability of death of a patient diagnosed with COVID-19, through variables such as age, sex, the presence of some comorbidities and other conditions.

\subsection{Variable selection and score construction}

A total of 35 variables were obtained from the database provided by Mexico. Of these, we found information related to the dates of onset of symptoms, date of registration in the system, date of death, as well as information related to the demographic location of cases, hospital care status, ICU admission, intubation and nationality. In addition, information was obtained regarding sex, pregnancy, smoking, and the presence of additional diseases and conditions such as asthma, pneumonia, diabetes, chronic obstructive pulmonary disease, immunosuppression, hypertension, cardiovascular disease, obesity, and chronic kidney disease. Finally, the database has an indicator of the patient's death. To begin the process of selection of variables, a time filter was carried out to obtain the cases of patients who had already passed 15 days after the contagion of the virus, and thus ensure that the patient is no longer infected with COVID-19. Of the acquired variables, the capacity of each one to affect the state of a person and to influence in the infection was evaluated, reason why it was decided to use 14 variables with information on the age, gender, smoking and previous diseases like asthma, pneumonia, diabetes, chronic obstructive pulmonary disease, immunosuppression, hypertension, cardiovascular disease, obesity, and chronic kidney disease. A machine learning model based on decision tree was chosen to predict the probability of death of a patient infected with COVID-19 from variables related to his clinical history. The design, 
training and testing of this model was done in Python. Some of the variables in the model were assigned as category variables to be better interpreted by the model. The design of the model was made with the Scikit-learn library, where the dataset was divided in a percentage of $80 \%$ for training, and $20 \%$ for testing. The model used was based on decision tree assemblies called LightGBM proposed by Ke. Guolin et al. (2017) [3]. Unlike other tree based models, this one has a very good computational efficiency. A search for hyperparameters was performed through a Bayesian optimization, through which the parameters that best train the model are found. The metrics used to evaluate the model efficiency was the AUC ROC. To ensure that the trained model works in general cases, it should adequately represent the probability distribution of each class, so a cross validation was performed where the data was divided into 3 shuffled folds in which the distribution of classes is preserved. The model with the best parameters was then used to build the COVID-19 risk calculator.

\section{Results}

\subsection{Characteristics of the Development Cohort}

The specific information on the patients used in the development set is specified in Table 1, where the distribution for each characteristic is differentiated for the groups of people who died from COVID-19 and those who did not die. The values for each of the characteristics in the table are as follows: (1) the condition is present, (2) condition is not present, (97) not applicable, (98) ignored, and (99) not specified.

\subsection{Predictor Selection}

Of the variables obtained from the database, the following were finally used to train the model: age, sex, pregnancy, smoking, asthma, pneumonia, diabetes, chronic obstructive pulmonary disease, immunosuppression, hypertension, cardiovascular disease, obesity, and chronic kidney disease. The variable to predict was the death of the patient. An analysis of the distribution of each one of the variables used for the training of the model was made, differentiating the populations by the variable to be predicted. See Figure 1. A model based on the LightGBM decision tree was trained with the above mentioned variables. The importance that each of these variables had for the model was evaluated using the Scikit-learn feature importance. See Figure 2.

PNEUMONIA

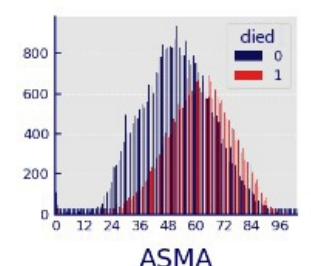

ASMA
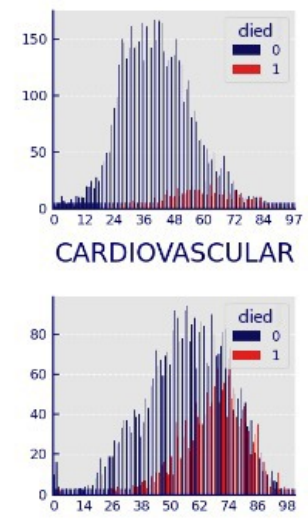

PREGNANCY
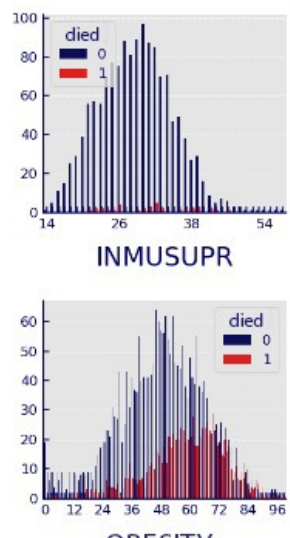

OBESITY

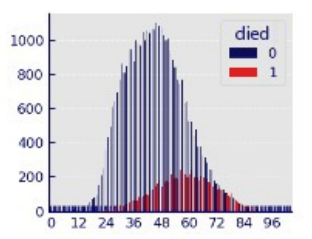

DIABETES

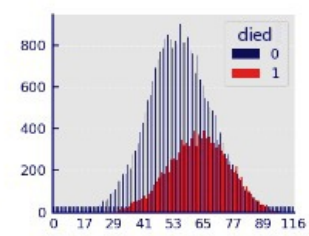

HYPERTENSION

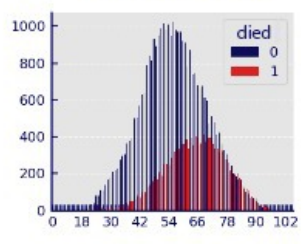

CHRONIC KIDNEY

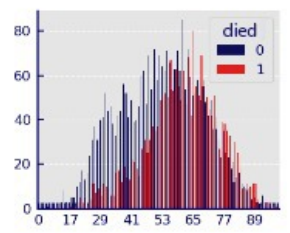

COPD

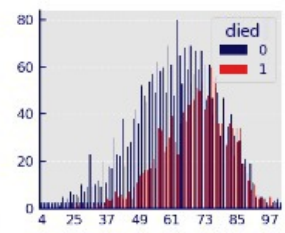

OTHER

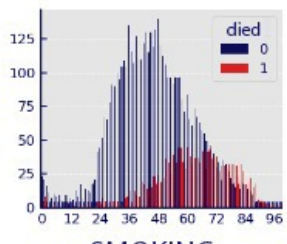

SMOKING

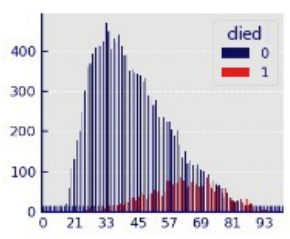

Figure 1: Histogram analysis of the variables used to train the model. 
A PREPRINT - OCTOBER 1, 2021

\begin{tabular}{|c|c|c|c|c|c|c|}
\hline & & Missing & Overall & Recovered & Deaths & P-Value \\
\hline $\mathrm{n}$ & & & 957532 & 864178 & 93354 & \\
\hline \multirow[t]{2}{*}{ Sex, n (\%) } & Female & 0 & $466996(48.8)$ & $433175(50.1)$ & $33821(36.2)$ & $<0.001$ \\
\hline & Male & & $490536(51.2)$ & 431003 (49.9) & $59533(63.8)$ & \\
\hline \multirow{3}{*}{ Pneumonia, n (\%) } & 1 & 0 & 161830 (16.9) & $93623(10.8)$ & $68207(73.1)$ & $<0.001$ \\
\hline & 2 & & $795693(83.1)$ & $770546(89.2)$ & $25147(26.9)$ & \\
\hline & 99 & & $9(0.0)$ & $9(0.0)$ & & \\
\hline \multirow[t]{4}{*}{ Pregnancy, n (\%) } & 1 & 0 & $6909(0.7)$ & $6816(0.8)$ & $93(0.1)$ & $<0.001$ \\
\hline & 2 & & $456667(47.7)$ & 423013 (48.9) & $33654(36.0)$ & \\
\hline & 97 & & $490536(51.2)$ & 431003 (49.9) & $59533(63.8)$ & \\
\hline & 98 & & $3420(0.4)$ & $3346(0.4)$ & $74(0.1)$ & \\
\hline \multirow[t]{3}{*}{ Diabetes, n (\%) } & 1 & 0 & $142071(14.8)$ & 105998 (12.3) & 36073 (38.6) & $<0.001$ \\
\hline & 2 & & $812365(84.8)$ & $755602(87.4)$ & $56763(60.8)$ & \\
\hline & 98 & & $3096(0.3)$ & $2578(0.3)$ & $518(0.6)$ & \\
\hline \multirow[t]{3}{*}{ COPD, n (\%) } & 1 & 0 & $13036(1.4)$ & $8521(1.0)$ & $4515(4.8)$ & $<0.001$ \\
\hline & 2 & & 941693 (98.3) & 853317 (98.7) & 88376 (94.7) & \\
\hline & 98 & & $2803(0.3)$ & $2340(0.3)$ & $463(0.5)$ & \\
\hline \multirow[t]{3}{*}{ Asthma, n (\%) } & 1 & 0 & $24184(2.5)$ & $22346(2.6)$ & $1838(2.0)$ & $<0.001$ \\
\hline & 2 & & $930576(97.2)$ & $839524(97.1)$ & $91052(97.5)$ & \\
\hline & 98 & & $2772(0.3)$ & $2308(0.3)$ & $464(0.5)$ & \\
\hline \multirow[t]{3}{*}{ Immunosuppression, $\mathrm{n}(\%)$} & 1 & 0 & $9867(1.0)$ & $7533(0.9)$ & $2334(2.5)$ & $<0.001$ \\
\hline & 2 & & $944735(98.7)$ & $854206(98.8)$ & $90529(97.0)$ & \\
\hline & 98 & & $2930(0.3)$ & $2439(0.3)$ & $491(0.5)$ & \\
\hline \multirow[t]{3}{*}{ Hypertension, n (\%) } & 1 & 0 & $180484(18.8)$ & $137884(16.0)$ & $42600(45.6)$ & $<0.001$ \\
\hline & 2 & & $774137(80.8)$ & $723861(83.8)$ & $50276(53.9)$ & \\
\hline & 98 & & $2911(0.3)$ & $2433(0.3)$ & $478(0.5)$ & \\
\hline \multirow[t]{3}{*}{ Cardiovascular disease, n (\%) } & 1 & 0 & $17890(1.9)$ & $12836(1.5)$ & $5054(5.4)$ & $<0.001$ \\
\hline & 2 & & $936793(97.8)$ & 848995 (98.2) & $87798(94.0)$ & \\
\hline & 98 & & $2849(0.3)$ & $2347(0.3)$ & $502(0.5)$ & \\
\hline \multirow[t]{3}{*}{ Obesity, n (\%) } & 1 & 0 & $164991(17.2)$ & $142342(16.5)$ & $22649(24.3)$ & $<0.001$ \\
\hline & 2 & & $789735(82.5)$ & $719498(83.3)$ & $70237(75.2)$ & \\
\hline & 98 & & $2806(0.3)$ & $2338(0.3)$ & $468(0.5)$ & \\
\hline \multirow[t]{3}{*}{ Chronic kidney disease, n (\%) } & 1 & 0 & $17384(1.8)$ & $10531(1.2)$ & $6853(7.3)$ & $<0.001$ \\
\hline & 2 & & 937345 (97.9) & $851321(98.5)$ & $86024(92.1)$ & \\
\hline & 98 & & $2803(0.3)$ & $2326(0.3)$ & $477(0.5)$ & \\
\hline \multirow[t]{3}{*}{ Smoking, n (\%) } & 1 & 0 & $70513(7.4)$ & $62996(7.3)$ & $7517(8.1)$ & $<0.001$ \\
\hline & 2 & & 884063 (92.3) & $798722(92.4)$ & 85341 (91.4) & \\
\hline & 98 & & $2956(0.3)$ & $2460(0.3)$ & $496(0.5)$ & \\
\hline \multirow[t]{3}{*}{ Other comorbidity, n (\%) } & 1 & 0 & $22160(2.3)$ & $17136(2.0)$ & $5024(5.4)$ & $<0.001$ \\
\hline & 2 & & $930490(97.2)$ & 843069 (97.6) & 87421 (93.6) & \\
\hline & 98 & & $4882(0.5)$ & $3973(0.5)$ & $909(1.0)$ & \\
\hline
\end{tabular}

Table 1: Characteristics of patients in the developmental cohort, for those who died from COVID-19 and those who did not. The values for each of the characteristics in the table are as follows: (1) the condition is present, (2) condition is not present, (97) not applicable, (98) ignored, and (99) not specified. 


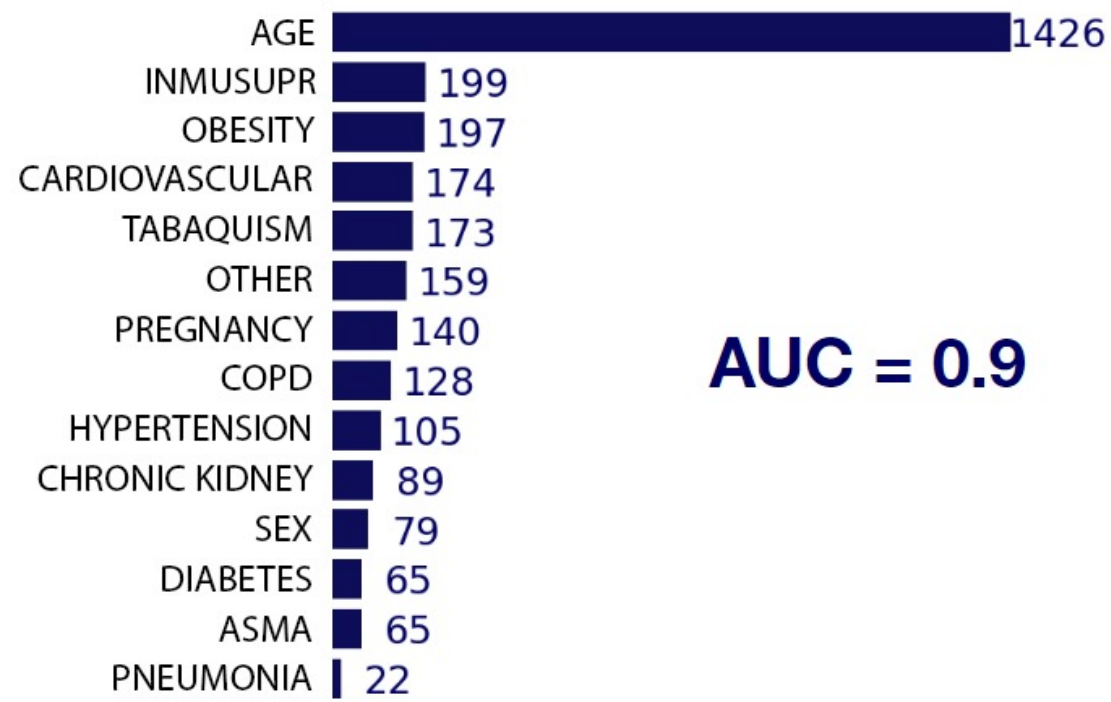

Figure 2: Importance of each feature for the designed model.

\subsection{Performance}

As mentioned above, the metric used to evaluate the performance of the model was the AUC ROC, because it is a commonly used measurement of the ability to distinguish between two classes. The model should adequately represent the probability distribution of each class, so a probability calibration with a sigmoid function was performed to improve the predictions. See Figure 3.
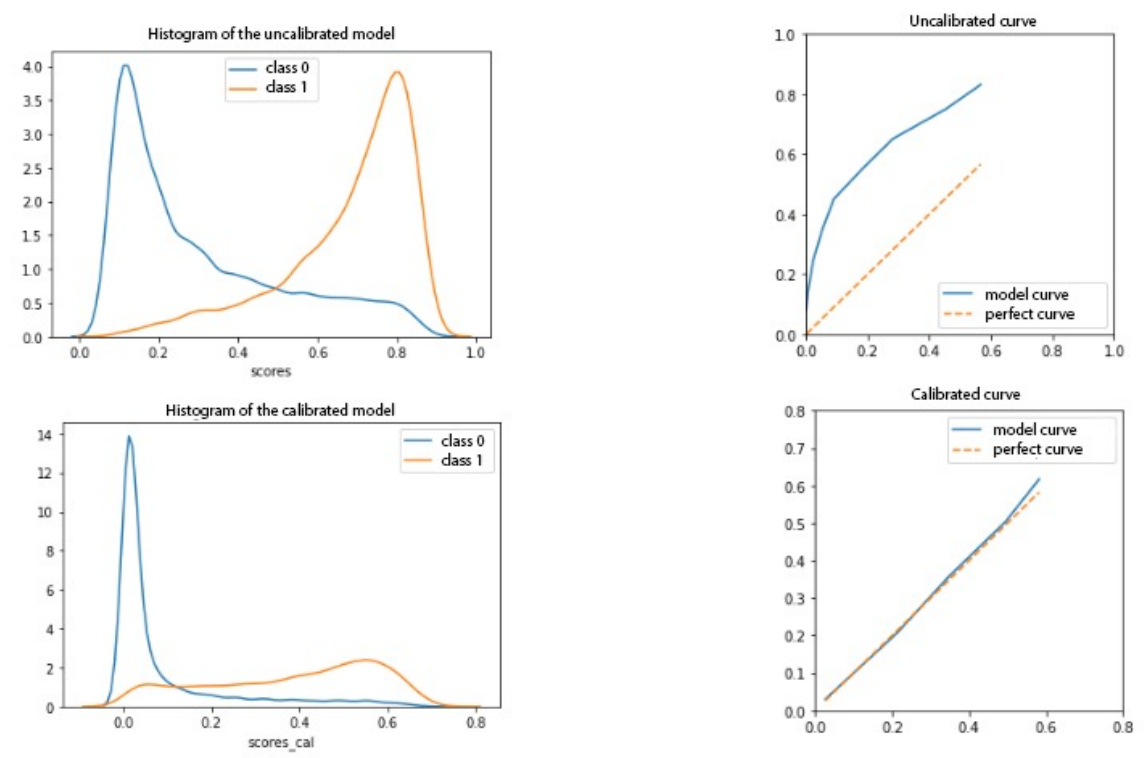

Figure 3: Calibration of the model.

Finally, after the whole process of preparation of the variables to be used, the training of the model by means of a crossed validation, the Bayesian optimization of hyperparameters, and finally the calibration of the model, an AUC of 0.89 was obtained. 


\subsection{Risk Calculator}

From the model that was developed to predict if a person is going to die from COVID-19, a calculator was developed in which when entering data about a person, an estimate of the probability of death from COVID-19 infection is given. For the use of the calculator a web application was made for both individuals and health professionals, through which a person's comorbidities are entered, and the probability of death is calculated using the trained balanced model. See Figure 4. The application can be accessed through the link: https://epidemiologia-matematica.org/IA/

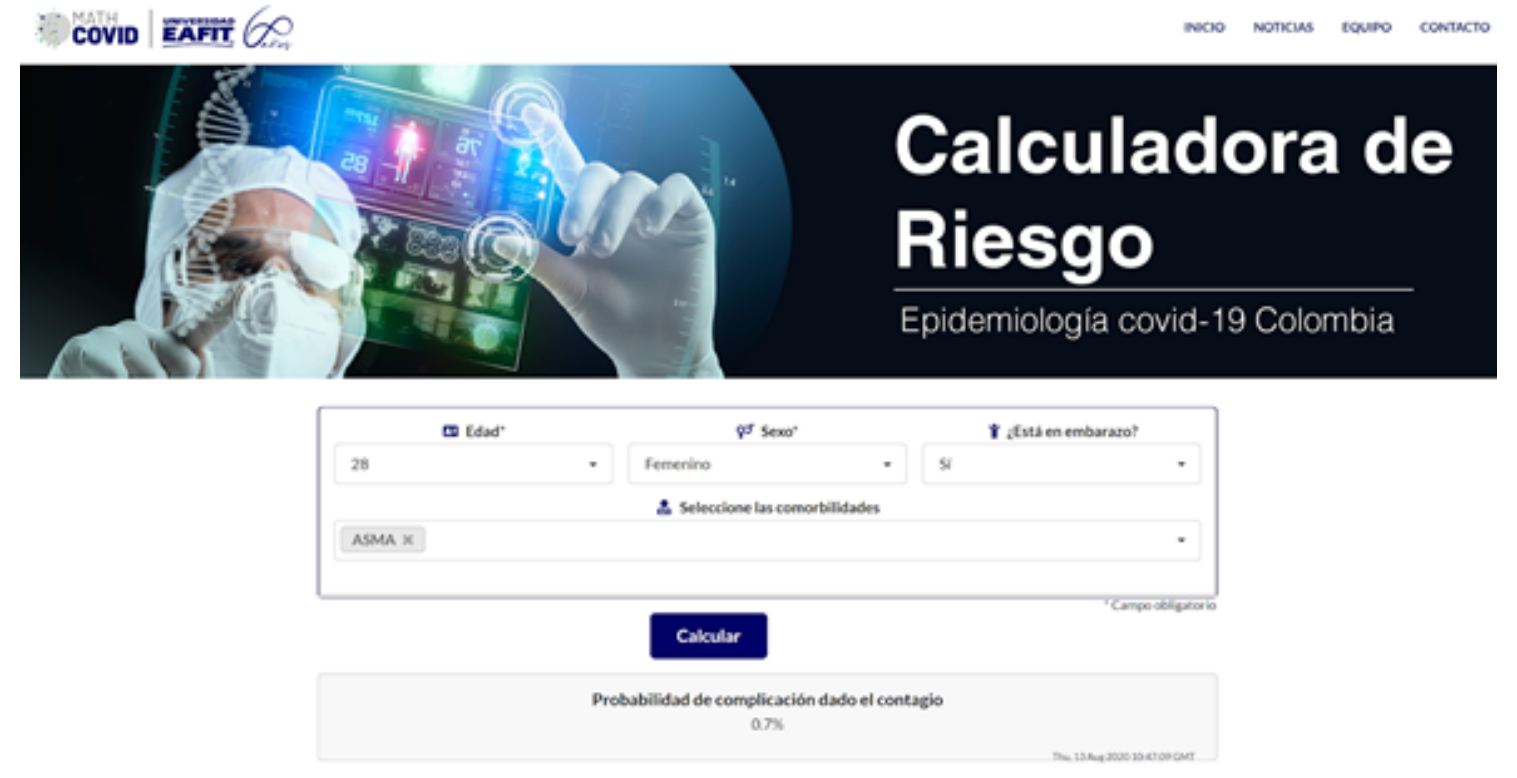

Figure 4: COVID-19 risk calculator.

\section{Discussion and Conclusions}

In this study a COVID-19 risk calculator was develop to determine the probability of death of a patient given specific information about the patient's medical conditions. The performance was evaluated using the AUC ROC metric and the result obtained was of 0.89 . The model proposed to predict the death of a person due to COVID-19 obtained a good result in the evaluated metrics, the implementation of verification methods used during the adaptation and processing of the data used to train the model was of great importance to ensure the reliability of the model, as well as the calibration of the model after being trained. The web application developed is easy to use and understand so we ensure that it can be used by anyone. During the development of the model a cross validation was evaluated, and finally the model was calibrated using a sigmoid function. To make use of the implemented model, a web application was developed to calculate the percentage of risk that a patient infected with COVID-19 may have according to their comorbidities, age and gender. This calculator is easy to use and is intended to help health care entities, organizations and individuals make decisions that can help during the COVID-19 pandemic.

\subsection{Future Work}

As future work we intend to use the data from the same source but after obtaining a greater amount of patients and important information, thus corroborating the functionality of the model and adjusting the aspects needed to improve the model. It is also important to use similar data from another country and evaluate the possibility of implementing a general application for any population. It is interesting to use the information collected in this study to develop a machine learning model to predict the critical situation of a patient, that is, if a patient is going to need care in a hospital, be intubated, or require oxygenation. In the same way, a calculator of probability of this risk can be developed.

A different model can be evaluated for hospitals, in which more specific information is used about each patient's medical history, monitoring of medical conditions such as oxygen saturation, blood type, pressure, among others. And in this way, make decisions that can help optimize processes within hospitals to make them more efficient. 


\section{Acknowledgements}

This research has been carried out in the framework of the project "Plataforma web para la recolección de datos, visualización, análisis, predicción y evaluación de estrategias de control de la enfermedad producida por SARS-CoV-2 mediante herramientas de modelación matemática, simulación e inteligencia artificial" which has been funded by the program MinCienciaTón (COVID-19 2020) of MinCiencias Colombia and EAFIT University through the agreement number 1216101576695.

\section{References}

[1] Amos Cahan et al. "Development and validation of a knowledge-driven risk calculator for critical illness in COVID-19 patients". In: The American journal of emergency medicine 39 (2020), pp. 143-145.

[2] Prateek K Gupta et al. "Development and validation of a risk calculator for prediction of cardiac risk after surgery". In: Circulation 124.4 (2011), pp. 381-387.

[3] Guolin Ke et al. "Lightgbm: A highly efficient gradient boosting decision tree". In: Advances in neural information processing systems 30 (2017), pp. 3146-3154.

[4] Wenhua Liang et al. "Development and validation of a clinical risk score to predict the occurrence of critical illness in hospitalized patients with COVID-19". In: JAMA internal medicine 180.8 (2020), pp. 1081-1089.

[5] Yufeng Shang et al. "Scoring systems for predicting mortality for severe patients with COVID-19". In: EClinicalMedicine 24 (2020), p. 100426. 